\title{
Autoimmune channelopathies: questions remain
}

\section{Jin Li and Ange Maguy}

We read with interest the Review by Lazzerini et al. (Autoimmune channelopathies as a novel mechanism in cardiac arrhythmias. Nat. Rev. Cardiol. http://dx.doi.org/10.1038/ nrcardio. $2017.61 ; 2017)^{1}$. In their manuscript, Lazzerini et al. provide a comprehensive overview of the current understanding of autoimmune-mediated cardiac arrhythmias. However, several aspects need to be clarified. Special focus is given to antibodies targeting cardiac ion channels, and the authors adopt the term "autoimmune cardiac channelopathies" (REF. 1), a designation first introduced by Li et al. in 2013 to define this disease entity ${ }^{2}$.

In Figures 1 and 3 and their respective legends, Lazzerini et al. illustrate and state that autoantibodies against $\mathrm{K}^{+}$voltage-gated channel subfamily KQT member 1 (KCNQ1) "enhance the slow component of the delayed rectifier $\mathrm{K}^{+}$current $\left(I_{\mathrm{Ks}}\right.$ )" (REF. 1). Furthermore, they claim that the anti-KCNQ1 autoantibodies "exerted an agonist-like electrophysiological effect on the KCNQ1 K+ channel”. Our concern is that anti-KCNQ1 autoantibodies per se have not been proven to be the mediators of the increase in $I_{\mathrm{Ks}}$. Previous data have shown that patient serum that contains anti-KCNQ1 autoantibodies increases $I_{\mathrm{Ks}}$ current density in human embryonic kidney (HEK) 293 cells expressing KCNQ1 and $\mathrm{K}^{+}$voltage-gated channel subfamily E member 1 (KCNE1) ${ }^{2}$, and that cardiac action potentials are shortened as a result of an increase in $I_{\mathrm{Ks}}$ in cardiomyocytes isolated from KCNQ1-immunized rabbits ${ }^{3}$. However, to date, no studies have been performed to establish a direct link between anti-KCNQ1 autoantibodies and an increase in $I_{\mathrm{Ks}}$.

Finally, Lazzerini et al. state that "anti$\mathrm{Na}_{\mathrm{v}} 1.5$ autoantibodies inhibiting $I_{\mathrm{Na}}$ were detected" (REF. 1). This claim constitutes an overstatement of the current literature, most probably arising from a misconception. Although a causative relationship between circulating anti- $\mathrm{Na}_{\mathrm{v}} 1.5$ autoantibodies and the observed electrophysiological effects is likely, such a relationship has not been demonstrated $^{4}$. From this perspective, we believe that Figures 1 and 2 and their respective legends illustrating anti- $\mathrm{Na}_{\mathrm{v}} 1.5$ antibody-mediated $I_{\mathrm{Na}}$ inhibition are misleading and overemphasize the current state of knowledge in this field.

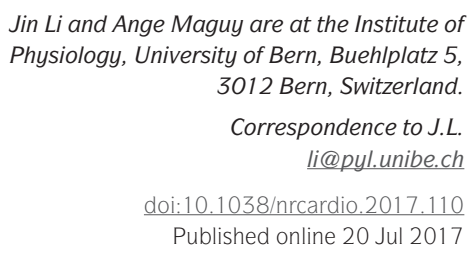

1. Lazzerini, P. E., Capecchi, P. L., Laghi-Pasini, F $\&$ Boutjdir, M. Autoimmune channelopathies as a nove mechanism in cardiac arrhythmias. Nat. Rev. Cardiol. http://dx.doi.org/10.1038/nrcardio.2017.61 (2017).

2. $\mathrm{Li}$, J. et al. Anti-KCNQ1 $\mathrm{K}^{+}$channel autoantibodies increase $I_{\mathrm{Ks}}$ current and are associated with QT interval shortening in dilated cardiomyopathy. Cardiovasc. Res. 98, 496-503 (2013).

3. Li, J. et al. Induced KCNQ1 autoimmunity accelerates cardiac repolarization in rabbits: potential significance in arrhythmogenesis and antiarrhythmic therapy. Heart Rhythm 11, 2092-2100 (2014).

4. Korkmaz, S. et al. Provocation of an autoimmune response to cardiac voltage-gated sodium channel $\mathrm{Na}_{\mathrm{v}} 1.5$ induces cardiac conduction defects in rats. J. Am. Coll. Cardiol. 62, 340-349 (2013).

Competing interests statement The authors declare no competing interests. 